\title{
Analysis of drug resistance in 1,861 strains of Acinetobacter baumannii
}

\author{
HAO JIN $^{1}$, FAN QIU ${ }^{2}$, HONG JIAN JI ${ }^{1}$ and QIANG LU ${ }^{1}$ \\ ${ }^{1}$ Department of Clinical Laboratory, Yancheng Third People's Hospital; ${ }^{2}$ Department of Pharmacy, \\ Yancheng Vocational Institute of Health Science, Yancheng, Jiangsu 224005, P.R. China
}

Received November 19, 2015; Accepted January 22, 2016

DOI: $10.3892 /$ br.2016.598

\begin{abstract}
Acinetobacter baumannii is an emerging human pathogen that causes hospital-acquired infections. The trend in increased antimicrobial resistance limits the choice of effective antimicrobial agents. The present study reports the resistance to Acinetobacter baumannii and analyzes the associations between antibiotic use and resistance rates at a general hospital between 2010 and 2014. A total of 1,861 isolates were obtained from clinical cultures, accounting for $10.33 \%$ of all detected bacteria $(1,861 / 18,016)$. The strains were mainly from respiratory samples $(1,628$ isolates, $87.5 \%)$ and the intensive care unit (696 isolates, 37.4\%). The resistance rates of Acinetobacter baumannii to the majority of antibiotics were $>50 \%$, particularly the resistance rate to cefoperazone/sulbactam increased from 47.37 in 2011 to $89.25 \%$ in 2014. However, the rates of imipenem and cilastatin sodium decreased from 81.03 to $69.44 \%$ due to the antibiotic policy. There were Pearson significant associations between the use of three antibiotics and resistance in Acinetobacter baumannii to this drug, piperacillin/tazobactam $(r=0.976$, $\mathrm{P}<0.01)$, gentamicin $(\mathrm{r}=0.870, \mathrm{P}<0.01)$ and cefoxitin $(\mathrm{r}=0.741$, $\mathrm{P}<0.05)$. Therefore, a combination of drugs should be adopted to treat Acinetobacter baumannii infections. Microbiology laboratory support and surveillance policies are essential to control the emergence of multidrug-resistance Acinetobacter baumannii.
\end{abstract}

\section{Introduction}

Acinetobacter baumannii is an emerging human pathogen that causes hospital-acquired infections (1). Acinetobacter baumannii infection could lead to a variety of illnesses such as pneumonia, meningitis and endocarditis (2). In recent years,

Correspondence to: Mrs. Fan Qiu, Department of Pharmacy, Yancheng Vocational Institute of Health Science, B3, 263 Jiefang South Road, Yancheng, Jiangsu 224005, P.R. China

E-mail: echo_qf@163.com

Key words: drug resistance, Acinetobacter baumannii, antibiotics usage the drug resistance rates of Acinetobacter baumannii are increasing quickly worldwide, particularly with the appearance of carbapenem-resistant Acinetobacter baumannii (3). Certain outbreaks of drug-resistant Acinetobacter baumannii have been documented previously $(4,5)$.

The trend in increased antimicrobial resistance limits the choice of effective antimicrobial agents. Multidrug-resistant Acinetobacter baumannii and pan-drug-resistant Acinetobacter baumannii have made numerous currently available antimicrobial drugs ineffective (2). Although several new antimicrobial therapeutics, such as the generation of nitric acid-producing nanoparticles (6), gallium maltolate treatment (7) and nanoemulsion (8), have been reported, the therapeutic efficacy and safety for people requires further investigation.

Currently, it is generally recognized that drug resistance is an unavoidable consequence of misuse and overuse of antibiotics. However, antibiotic treatments are not always the same for the differences of medical cognition in different regions, which leads to an eventual regional difference of bacterial resistance (9).

The purpose of the present study was to report the resistance to Acinetobacter baumannii, and analyze the association between antibiotic use and resistance rates at a general hospital in the east of China between 2010 and 2014.

\section{Materials and methods}

Bacterial isolates. The present study was performed at the Third People's Hospital, an 800-bed, tertiary-care teaching hospital affiliated with Southeast University (Yancheng, China). A total of 1,861 isolates were obtained from the clinical cultures between January 1, 2010 until December 31, 2014. Identification was performed using the VITEK 2 system (bioMerieux, Marcy l'Etoile, France) in the microbiological laboratory of the hospital.

Antimicrobial susceptibility. Antimicrobial susceptibility testing was performed using the disk diffusion method and susceptibility profiles were determined using zone diameter interpretive criteria, as recommended by the Clinical and Laboratory Standards Institute in 2011 (M100-S21). Mueller-Hinton agar (Oxoid, Thermo Fisher Scientific, Inc., Waltham, MA, USA) was used for all susceptibility tests. Escherichia coli American Type Culture Collection 
Table I. Resistance rates of Acinetobacter baumannii strains against antibiotics.

\begin{tabular}{lccccccrr}
\hline & \multicolumn{7}{c}{ Resistance rates, \% } \\
\cline { 2 - 8 } Antibiotics & $2011^{\mathrm{a}}$ & $2011^{\mathrm{b}}$ & $2012^{\mathrm{a}}$ & $2012^{\mathrm{b}}$ & $2013^{\mathrm{a}}$ & $2013^{\mathrm{b}}$ & $2^{2014^{\mathrm{a}}}$ & $2014^{\mathrm{b}}$ \\
\hline Cefoperazone/sulbactam & 47.37 & 56.19 & 74.26 & 86.71 & 99.46 & 99.61 & 87.69 & 89.25 \\
Ampicillin/sulbactam & 87.50 & 82.99 & 56.00 & 70.59 & 86.07 & 75.77 & 76.92 & 71.30 \\
Imipenem/cilastatin & 81.03 & 81.90 & 84.13 & 72.79 & 90.48 & 80.41 & 78.32 & 69.44 \\
Meropenem & 80.77 & 79.05 & 92.08 & 93.73 & 96.71 & 95.75 & 97.83 & 97.85 \\
Biapenem & 81.25 & 82.74 & 83.17 & 83.33 & 97.37 & 95.24 & 95.85 & 95.98 \\
Tetracycline & 73.00 & 75.00 & 85.33 & 99.25 & 99.74 & 99.32 & 99.74 & 99.75 \\
Tigecycline & - & - & - & - & 66.67 & 68.85 & 67.89 & 68.76 \\
Tobramycin & 83.33 & 60.00 & 40.00 & 72.79 & 89.52 & 74.23 & 70.63 & 69.44 \\
Amikacin sulfate & 78.00 & 88.57 & 83.00 & 79.95 & 69.88 & 65.68 & 62.10 & 60.09 \\
Gentamicin & 91.38 & 92.38 & 86.51 & 76.47 & 90.48 & 77.32 & 74.83 & 70.37 \\
Levofloxacin & 89.66 & 80.95 & 86.51 & 72.06 & 89.52 & 75.77 & 71.33 & 70.37 \\
Ciprofloxacin & 83.33 & 73.98 & 64.00 & 75.74 & 92.86 & 79.38 & 80.42 & 72.22 \\
Cefoxitin & 98.08 & 99.05 & 100.00 & 99.50 & 99.48 & 100.00 & 100.00 & 100.00 \\
Cefepime & 94.83 & 87.62 & 88.89 & 76.47 & 92.38 & 77.84 & 80.42 & 72.22 \\
Cefobutazine & 99.50 & 100.00 & 100.00 & 100.00 & 99.50 & 100.00 & 99.30 & 99.07 \\
Ceftazidime & 98.28 & 90.48 & 92.80 & 79.41 & 95.52 & 86.08 & 85.31 & 80.56 \\
Cefotaxime & 89.68 & 91.43 & 98.00 & 98.43 & 99.13 & 100.00 & 100.00 & 100.00 \\
Ceftriaxone & 83.33 & 89.74 & 96.00 & 94.12 & 99.52 & 100.00 & 99.30 & 99.07 \\
Aztreonam & 83.33 & 90.00 & 96.00 & 100.00 & 99.52 & 100.00 & 99.30 & 99.07 \\
Piperacillin/tazobactam & 86.21 & 85.71 & 81.60 & 75.00 & 90.48 & 78.35 & 79.72 & 71.30 \\
Compound sulfamethoxazole & 83.33 & 67.85 & 52.00 & 73.53 & 90.48 & 75.26 & 74.13 & 62.04 \\
Nitrofurantoin & 91.67 & 93.76 & 96.76 & 100.00 & 99.52 & 100.00 & 100.00 & 100.00 \\
\hline & & & & & & &
\end{tabular}

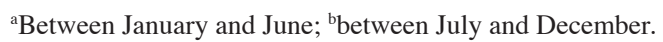

(ATCC) 25922, Escherichia coli ATCC 3518, Klebsiella pneumonia ATCC 700603, and Pseudomonas aeruginosa ATCC 7853 (all from ATCC, Manassas, VA, USA) were used as quality control strains for each batch of tests. Duplicate isolates, which were defined as repeated isolation, were of the same bacterial species for the same patients with the same profile of antibiotic susceptibility and they were excluded.

Antibiotic consumption. Antibiotic consumption data were collected from the hospital information system. The antibiotic usage was standardized based on the World Health Organization defined daily doses (DDDs) per 100 bed days (http://www.whocc.no/atc_ddd_index/).

Statistical analysis. A time series analysis model was used to analyze the association between the trend in quarterly antimicrobial consumption and the rates of resistance. SPSS software version 19.0 (IBM, Corp., Armonk, NY, USA) was used to calculate the Pearson correlation coefficient $(r, s)$. Statistical significance was two-sided and $\mathrm{P}<0.05$ was considered to indicate a statistically significant difference.

\section{Results}

Bacterial isolates. During the four years, 1,861 isolates were collected and identified as Acinetobacter baumannii, which represented $10.33 \%$ of all the isolated strains. The strains were cultured from respiratory samples $(1,628$ isolates, $87.5 \%)$, secretions and pus (84 isolates, $4.5 \%$ ), blood (51 isolates, $2.7 \%$ ), urine (44 isolates, $2.4 \%$ ), abdominal fluid (20 isolates, $1.1 \%$ ) and others (34 isolates, $1.9 \%$ ).

Changes in resistance to different antimicrobial agents. Table I summarizes the results of the susceptibility tests of Acinetobacter baumannii strains against antimicrobial agents. The majority of the rates of antimicrobial resistance in Acinetobacter baumannii were $>60 \%$ during the four years. Resistance to tobramycin, compound sulfamethoxazole, ampicillin/sulbactam and ciprofloxacin decreased significantly in 2011 due to the introduction of the antibiotic policy, a restriction of use of numerous antibiotics. Resistance to levofloxacin, cefepime, piperacillin/tazobactam, ceftazidime, imipenem/cilastatin and gentamicin decreased slowly in 2012. However, all of these rebounded in the first half of 2013, and subsequently decreased slowly thereafter.

Sulbactam is an irreversible inhibitor of $\beta$-lactamase, which is commonly used in the local hospital, and the increase of the resistance rates of cefoperazone/sulbactam was evident. There are three carbapenem antibiotics; imipenem, meropenem and biapenem. In 2011, the resistance rates of imipenem/cilastatin, meropenem and biapenem was almost $80 \%$, and three years later, the resistance rates of meropenem and biapenem increased 
Table II. Antibiotic administration between 2011 and 2014.

\begin{tabular}{|c|c|c|c|c|c|c|c|c|}
\hline Antibiotics & $2011^{\mathrm{a}}$ & $2011^{\mathrm{b}}$ & $2012^{\mathrm{a}}$ & $2012^{\mathrm{b}}$ & $2013^{\mathrm{a}}$ & $2013^{b}$ & $2014^{\mathrm{a}}$ & $2014^{\mathrm{b}}$ \\
\hline \multicolumn{9}{|l|}{ Cefoperazone/sulbactam } \\
\hline DDDs & 954.34 & $1,197.56$ & $1,363.88$ & $1,291.13$ & $1,350.35$ & $1,429.13$ & $1,853.25$ & $1,970.38$ \\
\hline DDD/100 patient-days & 1.94 & 2.18 & 2.46 & 2.23 & 0.44 & 0.46 & 0.63 & 0.63 \\
\hline \multicolumn{9}{|l|}{ Imipenem/cilastatin } \\
\hline DDDs & 204.35 & 242.42 & 224.22 & 226.14 & 263.75 & 260.75 & 314.01 & 328.75 \\
\hline DDD/100 patient-days & 0.42 & 0.44 & 0.40 & 0.41 & 0.09 & 0.08 & 0.11 & 0.11 \\
\hline \multicolumn{9}{|l|}{ Biapenem } \\
\hline DDDs & 789.61 & 810.41 & 852.25 & 699.50 & $1,676.25$ & 861.75 & 846.75 & $1,097.25$ \\
\hline DDD/100 patient-days & 1.61 & 1.47 & 1.54 & 1.20 & 0.55 & 0.28 & 0.28 & 0.35 \\
\hline \multicolumn{9}{|l|}{ Amikacin sulfate } \\
\hline DDDs & 224.71 & 240.10 & 265.80 & 285.00 & 348.50 & 333.40 & 186.00 & 192.60 \\
\hline DDD/100 patient-days & 0.46 & 0.44 & 0.48 & 0.49 & 0.19 & 0.11 & 0.06 & 0.06 \\
\hline \multicolumn{9}{|l|}{ Gentamicin } \\
\hline DDDs & $2,988.00$ & $3,022.00$ & $2,961.67$ & $2,777.80$ & $3,590.00$ & $2,011.80$ & $2,117.73$ & $1,559.94$ \\
\hline DDD/100 patient-days & 6.09 & 5.49 & 5.35 & 4.80 & 1.18 & 0.65 & 0.72 & 0.50 \\
\hline \multicolumn{9}{|l|}{ Levofloxacin } \\
\hline DDDs & $13,750.00$ & $12,966.00$ & $14,168.60$ & $12,068.00$ & $17,226.00$ & $13,628.20$ & $13,405.80$ & $13,240.80$ \\
\hline DDD/100 patient-days & 28.00 & 23.57 & 25.58 & 20.84 & 5.65 & 4.38 & 4.57 & 4.23 \\
\hline \multicolumn{9}{|l|}{ Cefoxitin } \\
\hline DDDs & $25,412.00$ & $23,035.00$ & $21,261.00$ & $16,384.33$ & $13,187.48$ & $8,907.67$ & $6,633.33$ & $4,614.67$ \\
\hline DDD/100 patient-days & 51.76 & 41.88 & 38.38 & 28.30 & 4.33 & 2.86 & 2.26 & 1.47 \\
\hline \multicolumn{9}{|l|}{ Cefepime } \\
\hline DDDs & 542.01 & 435.46 & 554.25 & 436.25 & 676.88 & 652.25 & 762.25 & 769.75 \\
\hline DDD/100 patient-days & 1.10 & 0.79 & 0.99 & 0.75 & 0.22 & 0.21 & 0.26 & 0.25 \\
\hline \multicolumn{9}{|l|}{ Ceftazidime } \\
\hline DDDs & $3,111.69$ & $2,741.32$ & $2,723.25$ & $2,232.75$ & $3,219.44$ & $2,280.00$ & $2,568.25$ & $2,815.00$ \\
\hline DDD/100 patient-days & 6.34 & 4.98 & 4.92 & 3.86 & 1.06 & 0.73 & 0.88 & 0.90 \\
\hline \multicolumn{9}{|l|}{ Piperacillin/tazobactam } \\
\hline DDDs & $2,940.30$ & $2,917.24$ & $2,480.92$ & $2,575.53$ & $6,233.70$ & $8,773.78$ & $8,148.18$ & $8,162.52$ \\
\hline DDD/100 patient-days & 5.99 & 5.30 & 4.48 & 4.55 & 2.05 & 2.82 & 2.78 & 2.59 \\
\hline
\end{tabular}

${ }^{\mathrm{a} B e t w e e n ~ J a n u a r y ~ t o ~ J u n e ; ~ ' b e t w e e n ~ J u l y ~ t o ~ D e c e m b e r . ~ D D D s, ~ d e f i n e d ~ d a i l y ~ d o s e s . ~}$

by $17 \%$, but the resistance rates of imipenem/cilastatin decreased by $14 \%$. Tigecycline was introduced in the hospital in 2013, and the resistance rate was $<70 \%$. Among a number of cephalosporin drugs, the third and the fourth generation cephalosporins, such as cefepime and ceftazidime, were active agents, but the other cephalosporins were not.

Association of hospital antibiotic use and resistance rate (\%) of Acinetobacter baumannii. The high level of antibiotic resistance was influenced by numerous factors; one of the most important factors was the overuse and misuse of antibiotics. Several antibiotic usage data were collected and were converted to DDDs, as shown in Table II. During the investigation period, the consumption of antibiotics varied from 192.60 to 25,412 DDDs. The usage of the second-generation cephalosporin drug ranked first, followed by quinolone, aminoglycoside and others.

There were statistically significant associations between the uses of several antibiotics and resistance in Acinetobacter
Table III. Association between the antibiotic use and resistance rate (\%) of Acinetobacter baumannii.

\begin{tabular}{lcc}
\hline Antibiotics & $\begin{array}{c}\text { Pearson } \\
\text { association }\end{array}$ & $\begin{array}{c}\text { P-value } \\
\text { (two-tailed) }\end{array}$ \\
\hline Cefoperazone/sulbactam & 0.601 & 0.115 \\
Imipenem/cilastatin & 0.601 & 0.115 \\
Biapenem & 0.097 & 0.097 \\
Amikacin sulfate & 0.138 & 0.744 \\
Gentamicin & $0.870^{\mathrm{a}}$ & 0.005 \\
Levofloxacin & 0.654 & 0.079 \\
Cefoxitin & $-0.741^{\mathrm{b}}$ & 0.035 \\
Cefepime & -0.324 & 0.433 \\
Ceftazidime & $0.748^{\mathrm{b}}$ & 0.033 \\
Piperacillin/tazobactam & $0.876^{\mathrm{a}}$ & 0.006 \\
\hline
\end{tabular}

${ }^{\mathrm{a}} \mathrm{P}<0.01 ;{ }^{\mathrm{b}} \mathrm{P}<0.05$. 
Table IV. Association between the use of various types of antibiotics and the resistance rates (\%) of Acinetobacter baumannii.

\begin{tabular}{lccc}
\hline & \multicolumn{2}{c}{ Resistance rates, \% } \\
\cline { 3 - 4 } Defined daily doses & Biapenem & Cefoperazone/sulbactam & Amikacin sulfate \\
\hline Aminoglycosides & & & 0.719 \\
Pearson association & $-0.924^{\mathrm{a}}$ & $-0.842^{\mathrm{b}}$ & 0.035 \\
P-value (two-tailed) & 0.009 & & 0.107 \\
Cephalosporins & & -0.505 & $0.875^{\mathrm{a}}$ \\
Pearson association & -0.785 & 0.307 & 0.004 \\
P-value (two-tailed) & 0.064 & & \\
\hline
\end{tabular}

${ }^{\mathrm{a}} \mathrm{P}<0.01 ;{ }^{\mathrm{b}} \mathrm{P}<0.05$.

baumannii to the drug as shown in Table III. The gentamicin usage was significantly correlated with resistance in Acinetobacter baumannii to this drug ( $\mathrm{rs}=0.870, \mathrm{P}<0.01$ ). Furthermore, the association between the use of various types of antibiotic and resistance rate (\%) of Acinetobacter baumannii was analyzed, as shown in Table IV. There were statistically significant associations between the resistance rate of biapenem and usage of aminoglycosides ( $\mathrm{r}=-0.924, \mathrm{P}<0.01)$.

\section{Discussion}

The drug resistance data between 2010 and 2014 showed that the resistance rates of the majority of antimicrobial drugs was $>70 \%$ in Acinetobacter baumannii. Thus, it became more difficult to cure Acinetobacter baumannii infection, and one more active agent is required based on the resistance experience. The antibiotic policy was introduced in the hospital in 2011, and the majority of antibiotics used were restricted and guided by rules. The policy promoted the rational use of antimicrobial drugs, and certain antimicrobial resistant rates periodically reduced. However, all the antibiotic resistance rates did not decrease at the same time to cure infection, and there was a fluctuation. These drops and fluctuations of the resistance rates of certain antibiotics were closely associated with the usage of antibiotics according to the statistical analysis, confirming that an antimicrobial susceptibility test is important in providing useful information for effective treatment, and occasionally more than one antibiotic is required to cure Acinetobacter baumannii infection. To decrease the spread of Acinetobacter baumannii infections, surveillance is also important to restrict the abuse of antibiotics and guide the rational usage of antibiotics.

\section{Acknowledgements}

The present study was supported by a project grant from Yancheng Medical Science and Technology Development Project (grant no. YK2013056).

\section{References}

1. Jones CL, Clancy M, Honnold C, Singh S, Snesrud E, Onmus-Leone F, McGann P, Ong AC, Kwak Y, Waterman P, et al: Fatal outbreak of an emerging clone of extensively drug-resistant Acinetobacter baumannii with enhanced virulence. Clin Infect Dis 61: 145-154, 2015.

2. Shields RK, Clancy CJ, Gillis LM, Kwak EJ, Silveira FP, Massih RC, Eschenauer GA, Potoski BA and Nguyen MH: Epidemiology, clinical characteristics and outcomes of extensively drug-resistant Acinetobacter baumannii infections among solid organ transplant recipients. PLoS One 7: e52349, 2012.

3. Snitkin ES, Zelazny AM, Montero CI, Stock F, Mijares L, Murray PR, Segre JA, Mullikin J, Blakesley R, Young A, et al; NISC Comparative Sequence Program: Genome-wide recombination drives diversification of epidemic strains of Acinetobacter baumannii. Proc Natl Acad Sci USA 108: 13758-13763, 2011.

4. Vilacoba E, Almuzara M, Gulone L, Rodriguez R, Pallone E, Bakai R, Centrón D and Ramírez MS: Outbreak of extensively drug-resistant Acinetobacter baumannii indigo-pigmented strains. J Clin Microbiol 51: 3726-3730, 2013.

5. Xu J, Sun Z, Li Y and Zhou Q: Surveillance and correlation of antibiotic consumption and resistance of Acinetobacter baumannii complex in a tertiary care hospital in northeast China, 2003-2011. Int J Environ Res Public Health 10: 1462-1473, 2013.

6. Mihu MR, Sandkovsky U, Han G, Friedman JM, Nosanchuk JD and Martinez LR: The use of nitric oxide releasing nanoparticles as a treatment against Acinetobacter baumannii in wound infections. Virulence 1: 62-67, 2010.

7. DeLeon K, Balldin F, Watters C, Hamood A, Griswold J, Sreedharan S and Rumbaugh KP: Gallium maltolate treatment eradicates Pseudomonas aeruginosa infection in thermally injured mice. Antimicrob Agents Chemother 53: 1331-1337, 2009.

8. Hwang YY, Ramalingam K, Bienek DR, Lee V, You T and Alvarez R: Antimicrobial activity of nanoemulsion in combination with cetylpyridinium chloride in multidrug-resistant Acinetobacter baumannii. Antimicrob Agents Chemother 57: 3568-3575, 2013.

9. Chen J, Li H, Yang J, Zhan R, Chen A and Yan Y: Prevalence and Characterization of Integrons in Multidrug Resistant Acinetobacter baumannii in Eastern China: A Multiple-Hospital Study. Int J Environ Res Public Health 12: 10093-10105, 2015. 